\title{
Percepção do impacto emocional da equipe de enfermagem diante a pandemia da COVID-19: revisão integrativa
}

Perception of the emotional impact of the nursing team in the face of the COVID-19 pandemic: an integrative review

Percepción del impacto emocional del equipo de enfermería ante la pandemia COVID-19: una revisión integradora

Bentinelis Braga da Conceição ORCID: https://orcid.org/0000-0003-1742-2384 Centro Universitário de Ciências e Tecnologia do Maranhão, Brasil

E-mail: bentinelisenfermeira@hotmail.com Annielson de Souza Costa ORCID: https://orcid.org/0000-0002-5487-7129 Universidade de São Paulo, Brasil E-mail: annielson.costa@usp.br

Monyka Brito Lima dos Santos ORCID: https://orcid.org/0000-0002-6866-9435

Universidade Federal do Ceará, Brasil E-mail: monyka.brito@ hotmail.com

Ingrid Loyane Bezerra Balata Silva ORCID: https://orcid.org/0000-0002-9412-5351

Universidade Federal do Maranhão, Brasil E-mail: ingridbalata19@gmail.com

Lívia Florêncio de Brito

ORCID: https://orcid.org/0000-0001-5324-8040 Universidade Federal do Piauí, Brasil E-mail: liviaflorbrito@gmail.com

Leila Dulce Guimarães Leal Alves ORCID: https://orcid.org/0000-0002-6456-0539 Instituto Camilo Filho, Brasil

E-mail: leila_dulce@hotmail.com

Caio Alexandre Costa Barbosa

ORCID: https://orcid.org/0000-0001-6732-1464 Centro Universitário de Ciências e Tecnologia do Maranhão, Brasil E-mail: caio1607@hotmail.com

Mariana Teixeira da Silva ORCID: https://orcid.org/0000-0002-1904-0921 Universidade Federal do Piauí, Brasil

E-mail: marytsilva44@gmail.com

Camylla Layanny Soares Lima ORCID: https://orcid.org/0000-0001-5015-6597

Faculdade Evangélica do Meio Norte, Brasil E-mail:enfcamyllasoares@gmail.com

Francisco Iago Fonseca Farias ORCID: https://orcid.org/0000-0003-4148-2360 Centro Universitário de Ciências e Tecnologia do Maranhão, Brasil E-mail: iagofarias621@gmail.com

Daniely Pereira de Sousa ORCID: https://orcid.org/0000-0001-5654-2616 Pontifícia Universidade Católica de Goiás, Brasil E-mail: dani.sousa09@gmail.com Luana de Oliveira ORCID: https://orcid.org/0000-0001-9678-1697 Christus Faculdade do Piauí, Brasil E-mail: luanabra@hotmail.com Ielda Pereira Rodrigues

ORCID: https://orcid.org/0000-0003-3365-4791 Centro Universitário UniFacid, Brasil E-mail: ieldapereira@hotmail.com 
Bárbara Mônica Lopes e Silva

ORCID: https://orcid.org/0000-0003-4969-3138 Centro Universitário de Ciências e Tecnologia do Maranhão, Brasil

E-mail: monicaalopees@gmail.com

Bárbara Maria Rodrigues dos Santos

ORCID: https://orcid.org/0000-0002-7335-2408 Universidade Federal do Piauí, Brasil E-mail: barbara.mariarss@gmail.com

Sara Kele Ramalho

ORCID: https://orcid.org/0000-0002-8017-9885 Universidade Estadual do Maranhão, Brasil E-mail: sararamalhorios@ hotmail.com

Erica Patrícia Dias de Sousa

ORCID: https://orcid.org/0000-0001-7675-902X Unidades Integradas de Pós-graduação, Brasil E-mail: ericaigsousa@gmail.com

Cleonilma dos Santos Coelho

ORCID: https://orcid.org/0000-0002-2609-7117

Universidade Estadual do Maranhão, Brasil E-mail: cleo_maninha@hotmail.com

Adriano Nogueira da Cruz

ORCID: https://orcid.org/0000-0002-3572-6878

Faculdade Internacional SIGNORELLI, Brasil E-mail: adrianonogueira.cx@gmail.com

Lívia Martins Dantas

ORCID: https://orcid.org/0000-0002-2286-4366 Universidade Federal do Piauí, Brasil E-mail: livia_m_dantas@hotmail.com

Paula Lima de Mesquita

ORCID: https://orcid.org/0000-0001-6198-4590 Centro Universitário UniFacid, Brasil

E-mail: paulinha.demesquita@gmail.com

\section{Resumo}

A pandemia da COVID-19 pode ser descrita como uma dessas crises, a qual tem se caracterizado como um dos maiores problemas de saúde pública internacional das últimas décadas, tendo atingido praticamente todo o planeta. Um evento como esse ocasiona perturbações psicológicas e sociais que afetam a capacidade de enfrentamento de toda a sociedade, em variados níveis de intensidade e propagação. O presente trabalho tem como objetivo abordar a percepção da equipe de enfermagem diante da pandemia de coronavírus, assim como, apresentar evidências científicas sobre fatores associados ao impacto ocupacional e psicológico provocado por elas sobre os profissionais da saúde. O método utilizado na presente pesquisa foi a análise bibliográfica do tipo integrativa abordando a temática, sendo realizada através da biblioteca virtual em saúde utilizando como descritores: Enfermagem. Coronavírus. Pandemia. Saúde Mental. Observou-se com estudo que as equipes de enfermagem têm atuado constantemente sob pressão psicológica, podendo ocasionar depressão e até mesmo a ansiedade. Através dos estudos realizados, concluise que, Diante de uma pandemia como a que vivemos, os profissionais da enfermagem constituem parte de um dos grupos mais afetados, expostos a dor emocional e ao risco de contágio que afeta consideravelmente a saúde mental. Entretanto, cuidados imediatos devem ser aplicados, em todos os níveis e pelas mais distintas áreas de conhecimento, com a finalidade de reduzir resultados ainda mais negativos na saúde mental destes profissionais.

Palavras-chave: Enfermagem; Coronavírus; Pandemia; Saúde mental.

\section{Abstract}

The COVID-19 pandemic can be described as one of these crises, which has been characterized as one of the biggest international public health problems of the last decades, having affected practically the entire planet. Such an event causes psychological and social disturbances that affect the coping capacity of the whole society, at varying levels of intensity and spread. The present study aims to address the perception of the nursing team in the face of the coronavirus pandemic, as well as to present scientific evidence on factors associated with the occupational and psychological impact caused by them on health professionals. The method used in the present research was the bibliographic analysis of the integrative type addressing the theme, being carried out through the virtual health library using as descriptors: Nursing. Coronavirus. Pandemic. Mental health. It was observed with a study that the nursing teams have been acting constantly under psychological pressure, which can cause depression and even anxiety. Through the studies carried out, it is concluded that, in the face of a pandemic such as the one we are experiencing, nursing professionals are part of one of the most affected groups, exposed to emotional pain and the risk of contagion that considerably affects mental health. However, immediate care must be applied, at all levels and by the most different areas of knowledge, in order to reduce even more negative results in the mental health of these professionals. Keywords: Nursing; Coronavirus; Pandemic; Mental health. 


\begin{abstract}
Resumen
La pandemia de COVID-19 puede describirse como una de estas crisis, que se ha caracterizado como uno de los mayores problemas de salud pública internacional en las últimas décadas, habiendo afectado prácticamente a todo el planeta. Un evento como este provoca perturbaciones psicológicas y sociales que afectan la capacidad de afrontamiento de toda la sociedad, en distintos niveles de intensidad y extensión. Este estudio tiene como objetivo abordar la percepción del personal de enfermería ante la pandemia de coronavirus, así como presentar evidencia científica sobre los factores asociados al impacto ocupacional y psicológico que provocan en los profesionales de la salud. El método utilizado en esta investigación fue el análisis bibliográfico de tipo integrativo, abordando la temática, siendo realizado a través de la biblioteca virtual en salud utilizando los siguientes descriptores: Enfermería. Coronavirus. Pandemia. Salud mental. En un estudio se observó que los equipos de enfermería han estado actuando constantemente bajo presión psicológica, lo que puede provocar depresión e incluso ansiedad. A través de los estudios realizados se concluye que, ante una pandemia como la que estamos viviendo, los profesionales de enfermería forman parte de uno de los grupos más afectados, expuestos al dolor emocional y al riesgo de contagio que afecta considerablemente la salud mental. Sin embargo, se debe aplicar una atención inmediata, a todos los niveles y por las más diversas áreas de conocimiento, con el fin de reducir aún más los resultados negativos en la salud mental de estos profesionales.
\end{abstract}

Palabras clave: Enfermería; Coronavirus; Pandemia; Salud mental.

\title{
1. Introdução
}

O problema proposto neste artigo é de extrema importância, pois visa abordar a percepção da equipe de enfermagem diante da pandemia de coronavírus, assim como, apresentar evidências científicas sobre fatores associados ao impacto ocupacional e psicológico provocado por elas sobre os profissionais da saúde.

O COVID-19 foi descoberto em 31 de dezembro de 2019 em Wuhan, China e disseminou-se aceleradamente tornando-se uma pandemia. O vírus é altamente patogênico e causa infecções do trato respiratório como a Síndrome Respiratória Aguda Grave (SRAG) e pode levar ao óbito. É transmitido através de contato direto com gotículas de saliva produzidas através da fala, tosse e espirros de um indivíduo contaminado. Além do mais, pode-se contrair o vírus ao tocar o rosto (olhos, nariz e boca) após o contato com superfícies e objetos contaminados (Portugal et al., 2020).

Atualmente, a pesquisa sobre o SARS-CoV-2 ainda se encontra em estágio inicial. Há pouco conhecimento sistematizado sobre a epidemiologia, características clínicas, diagnóstico, tratamento e prevenção da COVID-19, o que aumenta a insegurança dos trabalhadores de saúde que estão diretamente expostos, devido ao contato com indivíduos infectados. O SARS-CoV-2 apresenta características específicas (estrutura genética e mecanismos patogênicos) que impõem grandes desafios para a prevenção e tratamento da infecção, o que pode impactar diretamente a saúde mental dos profissionais que cuidam das pessoas infectadas. A forma grave de apresentação da COVID-19 cursa com síndrome respiratória aguda grave. Os pacientes que desenvolvem essa forma podem evoluir rapidamente a óbito (Oliveira et al., 2020).

A COVID-19 foi registrada em mais de 180 países ao redor do mundo, e mediante ao grande avanço da contaminação da doença, várias autoridades governamentais vêm adotando diversas estratégias, com a intenção de reduzir o ritmo da progressão da doença. Atualmente, pesquisadores e profissionais da área da saúde estão em um constante desafio conforme o avanço no número de casos de COVID-19, pois a doença ainda não possui o risco clínico totalmente definido, como também não se conhece com exatidão o padrão de transmissibilidade, infectividade, letalidade e mortalidade. Ressalta-se que ainda não há vacinas ou medicamentos específicos disponíveis contra a doença (Pereira et al., 2020).

Nesse cenário, considera-se que o enfermeiro por encontrar-se na linha de frente da assistência de combate à pandemia do novo coronavírus, estão suscetíveis de serem acometidas por razões psicoemocionais como a ansiedade, o medo, a insônia e depressão, além, da probabilidade da percepção de esgotamento físico e mental, perda de distanciamento emocional e sentido por motivo de realização das suas atividades de atendimentos em unidades de saúde hospitalares ou ambulatoriais com pacientes teoricamente contaminados pelo vírus (Brito et al., 2021). 
A preocupação com a saúde mental da população se intensifica durante uma grave crise social. A pandemia da COVID-19 pode ser descrita como uma dessas crises, a qual tem se caracterizado como um dos maiores problemas de saúde pública internacional das últimas décadas, tendo atingido praticamente todo o planeta. Um evento como esse ocasiona perturbações psicológicas e sociais que afetam a capacidade de enfrentamento de toda a sociedade, em variados níveis de intensidade e propagação (Faro et al., 2020).

Os profissionais de saúde estão em alto risco de adquirir COVID-19 devido à exposição a pacientes e principalmente pela disponibilidade limitada de Equipamentos de Proteção Individual (EPI), falta de treinamento adequado para o enfrentamento de surtos de doenças altamente infecciosas e uso inadequado de EPI. É imprescindível ressaltar que muitos profissionais de saúde foram infectados e vários perderam a vida desde o início da pandemia (Portugal et al., 2020).

Diante destes acontecimentos, as equipes de saúde estão sob constante pressão psicológica. Entre esses profissionais, destacam-se as equipes de enfermagem, tendo importante papel na saúde pública no controle e prevenção de infecção. Em todo mundo, a enfermagem tem trabalhado sob constante pressão, combatendo não apenas o vírus, mas diversas dificuldades impostas sobretudo pelo risco de infecção e pela escassez de proteção.

\subsection{Contexto de Pandemia e repercussões na saúde mental}

Maior parte das pessoas infectadas pode ser assintomática ou apresentarem sintomas leves a moderados, semelhante ao estado gripal. As manifestações mais frequentes são: tosse, febre e falta de ar. Na forma mais severa, o quadro clínico pode manifestar: pneumonia pulmonar, síndrome respiratória aguda grave, alterações, hematológicas edema pulmonar e de coagulação e até mesmo falência múltipla de órgãos (Ferreira et al., 2021).

Dados do relatório da OMS, publicado em 08 de maio de 2020, estão documentados 3.759 .967 casos confirmados, 259.474 mortes, com registro em países de todas as regiões do mundo. O primeiro caso da doença no Brasil, foi comunicado em 25 de fevereiro de 2020 e o número de comprometidos pelo vírus tem aumentado, desde esse momento, gradativamente. $\mathrm{O}$ Brasil constatou 145.328 casos e 9.897 mortes até o dia 08 de maio de 2020 (Duarte et al., 2020).

O cuidado é para a enfermagem a essência de suas práticas e o aspecto predominante que a distingue das demais profissões na área da saúde, definida como arte, técnica, intuição e sensibilidade. Cuidar de toda a complexidade humana constitui-se para o enfermeiro um desafio, pois suas demandas nunca cessam e nem poderão ser atendidas por completo. Durante o processo de adoecimento, quando surgem fragilidades, medos, ansiedades e desconfortos, a atenção à dimensão emocional do ser humano se faz mais necessária ainda (Humerez et al., 2020).

A assistência de enfermagem, além de solicitar prática científica e técnica, emprega medidas de controle emocional, frente a assistência ao cuidado que possibilita. Contudo, os desgastes emocionais e físicos afetam nas atividades laborais e são capazes de ocasionar em prejuízos tanto na saúde desse profissional, quanto de pacientes que estão sob seus cuidados (Rosa et al., 2021).

A situação de a pandemia expor o enfermeiro a uma circunstância complexa, muito dinâmica e inesperada é capaz de impossibilitar o seu bem-estar emocional e a sua adaptação e, em consequência, a prestação de cuidados (Diogo et al., 2021).

Lamentavelmente, no meio de fragilidades destacadas em um contexto nada equilibrado, outro aspecto tornou-se alarmante: a saúde mental dos profissionais de enfermagem. Apesar de a resposta a COVID-19 até o momento atual tenha se concentrado primordialmente em abranger a disseminação e prevenir a mortalidade, a pandemia apresentou também, que dispõe potencial para criar uma crise de sofrimento psicológico de enorme repercussão no sistema de saúde mental (RamosToescher et al., 2021).

Estudos sobre implicações na saúde mental em decorrência da pandemia do novo coronavírus ainda são escassos, por se tratar de fenômeno recente, mas apontam para repercussões negativas importantes. Além disso, pesquisas anteriores sobre 
outros surtos infecciosos revelaram desdobramentos desadaptativos, em curto, médio e longo prazo, para a população geral e para os profissionais da saúde (Schmidt et al., 2020).

No Brasil, ainda não há dados oficiais sobre o número de profissionais de saúde acometidos pela COVID-19. Como iniciativa do Conselho Federal de Enfermagem, foi desenvolvido um observatório no qual os profissional de enfermagem podem relatar seu adoecimento. Até o dia 13 de abril de 2020, foram registrados 1.203 casos de adoecimento e 18 óbitos. Acredita-se que esse número seja subestimado, por ser uma notificação voluntária. Esses dados demonstram a necessidade de ações protetivas que permitam o cuidado integral à saúde do profissional de enfermagem, com a implementação de protocolos que possam reduzir os riscos de contaminação durante a atividade laboral (Miranda et al., 2020).

A pandemia da COVID-19 está ocasionando um prejuízo enorme para indivíduos, famílias, comunidades e sociedades em todo o mundo. A vida cotidiana mudou profundamente, a economia entrou em recessão e muitas das redes tradicionais de segurança social, econômica e de saúde pública nas quais as pessoas confiam em tempos difíceis foram submetidas a pressões sem precedentes. A OMS observa que os trabalhadores da enfermagem pressionados com essa situação apresentam altos níveis de ansiedade, acrescidos do risco de adoecer, provocando severos problemas de saúde mental e aumentando os casos da Síndrome de Burnout, além de gerar ansiedade, depressão e estresse associado (Humerez, Ohl \& Silva, 2020).

As repercussões psicológicas nos integrantes da equipe de Enfermagem constituem consequências negativas para os países. As pressões extremas sofridas pelos Enfermeiros no decorrer da pandemia é capaz de aumentar o risco de burnout, o que impacta fortemente na vida profissional e pessoal dos membros da equipe (Kirby et al., 2020).

De acordo com Esperidião, Saidel e Rodrigues (2020) sobre a dimensão das condições laborais, essas, por vezes, colaboram para a pressão psicológica e sintomas psicossomáticos nos profissionais de saúde destacando-se: sobrecarga de trabalho; equipamentos e suporte organizacional escassos; política frágil de cargos e salários; inexistência de piso salarial da categoria; elevada carga horária; baixa remuneração; duplos vínculos empregatícios; vínculos precários nos contratos de trabalho; responsabilidade elevada; lida cotidiana com a dor, sofrimento, morte.

Os elevados níveis de estresse manifestam uma séria ameaça à saúde mental dos profissionais, aumentando as taxas de depressão, ansiedade, transtorno de estresse pós-traumático (TEPT) e comportamentos negativos o que é capaz de comprometer a efetividade da jornada de trabalho dos profissionais da saúde (Bezerra et al., 2020).

É de grande importância que os enfermeiros chefes, possuam um olhar amplificado para identificar os colaboradores que ache-se passando por essa situação e dessa forma ofereçam apoio, visto que, havendo uma liderança que comanda com um comprometimento afetivo, com empatia, escuta ativa ela é capaz de acolher esse profissional que precisa de cuidado, conduzindo e incentivando a procurar ajuda de um especialista (Nunes et al., 2020).

Disseminação de informações de fontes verdadeiras, com clareza, transparência e responsabilidade é uma indispensável estratégia neste momento de pandemia, haja vista que as informações da COVID-19 são dinâmicas e modificam a todo momento, é indispensável positiva comunicação para que os profissionais sejam capazes de ser atualizados das medidas de prevenção e controle facilitando a assistência de enfermagem de qualidade, assim como segurança para o profissional (Gomes et al., 2020).

De acordo com Miranda et al. (2020) embora medidas preventivas sejam tomadas, os profissionais de saúde vivenciam situações sem precedentes, tendo que tomar decisões difíceis que podem ocasionar agravos psicológicos a longo prazo, causados por danos morais. Assim, é vital que os governantes e gestores reconheçam o valor desses profissionais e os tratem com humanidade, garantindo sua segurança e saúde. Em uma tentativa de minimizar as consequências deste agravo na saúde física e mental dos profissionais, observa-se nas mídias sociais e em canais oficiais a solidariedade de especialistas em saúde mental na oferta de apoio emocional e orientações sobre hábitos saudáveis, visando à manutenção da saúde desses profissionais. 


\section{Metodologia}

O estudo consiste em uma revisão de literatura do tipo integrativa (Figura 1) sobre a Percepção do impacto emocional da equipe de enfermagem diante a pandemia da COVID-19, sendo que este estudo ainda permitiu refletir sobre a necessidade de garantir uma comunicação clara e informativa sobre estratégias para redução desses sintomas de sofrimento psíquico, além de fornecer o fundamental suporte psicológico e social para esses indivíduos em vulnerabilidade. Continuamente foram realizadas buscas nas bases de dados LILACS, MEDLINE, BDENF e SCIELO, usando os seguintes descritores no idioma português, conforme o DECS (Descritores em Ciências da Saúde): Enfermagem. Coronavírus. Pandemia. Saúde Mental.

Para a análise dos dados pertinentes à temática deste estudo, foram definidos previamente por critérios de inclusão: artigos escritos nos idiomas português, tempo do artigo (artigos publicados em 2020), e disponíveis para acesso na íntegra. Dessa forma, preconizou-se por excluir os artigos de textos incompletos, resumos, teses, artigos que fogem à temática e artigos duplicados.

A revisão integrativa, finalmente, é a mais ampla abordagem metodológica referente às revisões, permitindo a inclusão de estudos experimentais e não-experimentais para uma compreensão completa do fenômeno analisado. Combina também dados da literatura teórica e empírica, além de incorporar um vasto leque de propósitos: definição de conceitos, revisão de teorias e evidências, e análise de problemas metodológicos de um tópico particular (Souza, Silva \& Carvalho, 2010).

Figura 1. Esquema das etapas de uma revisão integrativa.

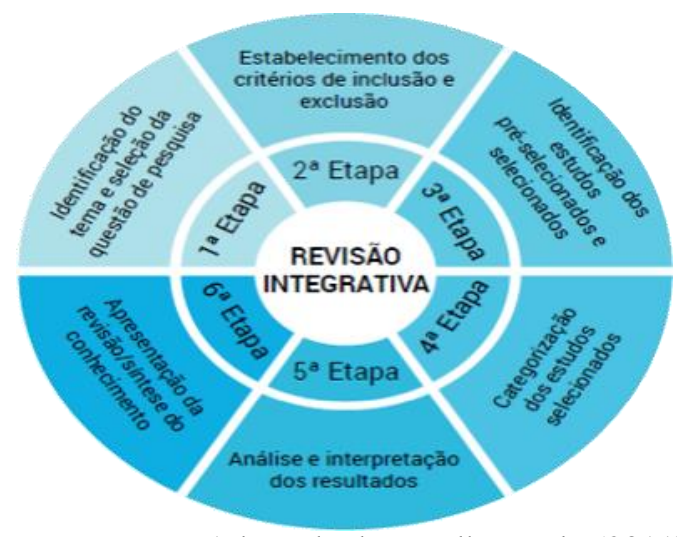

Fonte: Adaptado de Botelho et al., (2011).

Foram examinadas as referências de artigos selecionados para identificar aqueles que não serão cobertos pela busca. Finalizada a coleta de dados, foi realizada uma análise dos objetivos e resultados de todos os estudos com o propósito de obter os pontos de partida e desfechos que mais fundamentavam o tema pesquisado.

As etapas metodológicas usadas para a seleção dos estudos são apresentadas abaixo (Quadro 1). 
Quadro 1. Etapas Metodológicas usadas para seleção e inclusão do estudo.

\begin{tabular}{|c|c|c|c|c|}
\hline SCIELO: 16 & LILACS: 32 & MEDLINE: 174 & BDENF: 36 & Total: 258 \\
\hline Artigos Excluídos & \multicolumn{4}{|c|}{$(\mathrm{N}=138)$} \\
\hline $\begin{array}{c}\text { Artigos selecionados } \\
\text { para avaliação e leitura } \\
\text { integral }\end{array}$ & \multicolumn{4}{|c|}{$(\mathrm{N}=112)$} \\
\hline $\begin{array}{c}\text { Artigos incluídos para } \\
\text { análise dos dados deste } \\
\text { estudo }\end{array}$ & \multicolumn{4}{|c|}{$(\mathrm{N}=08)$} \\
\hline
\end{tabular}

Fonte: Autores (2020).

\section{Resultados e Discussão}

Nesta revisão integrativa foram incluídos 08 artigos. Nas bases de dados foram encontrados 258 artigos, destes, 16 foram no SCIELO, 32 na LILACS, 36 na BDENF e 174 na MEDLINE. Excluiu-se 250 por não atenderem os critérios propostos: artigos completos referentes à pesquisa (Percepção do impacto emocional da equipe de enfermagem diante a pandemia da COVID-19), idioma (português), tempo do artigo (artigos publicados em 2020), tipo de estudo (artigos), base de dados (LILACS, MEDLINE BDENF e SCIELO) e artigos duplicados.

Ano de publicação, todos foram publicados em 2020. Quanto ao tipo de estudo, cinco são revisões bibliográficas, dois estudos pilotos de um ensaio clínico prospectivo randomizado e um estudo relato de experiência (Quadro 2).

Quadro 2. Distribuição sinóptica demonstrativa dos estudos quanto ao Autor, título do artigo, objetivo do estudo e Conclusão.

\begin{tabular}{|c|c|c|c|c|}
\hline & Autor & Título do artigo & Objetivo & Conclusão \\
\hline A1 & $\begin{array}{l}\text { Schmidt et al. } \\
(2020)\end{array}$ & $\begin{array}{c}\text { Saúde mental e } \\
\text { intervenções } \\
\text { psicológicas diante da } \\
\text { pandemia do novo } \\
\text { coronavírus (COVID- } \\
\text { 19). }\end{array}$ & $\begin{array}{l}\text { Sistematizar conhecimentos } \\
\text { sobre implicações na saúde } \\
\text { mental e intervenções } \\
\text { psicológicas diante da } \\
\text { pandemia do novo } \\
\text { coronavírus. }\end{array}$ & $\begin{array}{l}\text { Nesse sentido, sugerem-se levantamentos } \\
\text { sobre implicações na saúde mental diante da } \\
\text { pandemia e sobre intervenções psicológicas } \\
\text { alinhadas às necessidades do contexto } \\
\text { brasileiro, considerando as características de } \\
\text { diferentes populações atingidas pela COVID- } \\
19 \text { e, em particular, de pessoas e grupos em } \\
\text { maior vulnerabilidade socioeconômica. } \\
\text { Entende-se que, embora imponha desafios } \\
\text { adicionais à atuação dos psicólogos no Brasil } \\
\text { e no mundo, a pandemia do novo coronavírus } \\
\text { pode contribuir para o aperfeiçoamento da } \\
\text { prática e da pesquisa em situações de crise, } \\
\text { emergência e desastre. }\end{array}$ \\
\hline $\mathrm{A} 2$ & Faro et al. (2020) & $\begin{array}{l}\text { COVID-19 e saúde } \\
\text { mental: A emergência } \\
\text { do cuidado. }\end{array}$ & $\begin{array}{l}\text { Buscou reunir informações e } \\
\text { achados de pesquisa a } \\
\text { respeito do impacto de tais } \\
\text { crises na saúde mental. }\end{array}$ & $\begin{array}{l}\text { Portanto, esforços imediatos devem ser } \\
\text { empregados, em todos os níveis e pelas mais } \\
\text { diversas áreas de conhecimento, a fim de } \\
\text { minimizar resultados ainda mais negativos na } \\
\text { saúde mental da população. Cabe, enfim, } \\
\text { investir em adequada assistência à saúde e, } \\
\text { sobretudo, na ciência em geral, para que esse } \\
\text { período seja abreviado e que os profissionais } \\
\text { de saúde estejam capacitados para os desafios } \\
\text { do cuidado. }\end{array}$ \\
\hline
\end{tabular}




\begin{tabular}{|c|c|c|c|c|}
\hline A3 & $\begin{array}{l}\text { Oliveira et al. } \\
\qquad(2020)\end{array}$ & $\begin{array}{l}\text { Impactos psicológicos } \\
\text { e ocupacionais das } \\
\text { sucessivas ondas } \\
\text { recentes de pandemias } \\
\text { em profissionais da } \\
\text { saúde: revisão } \\
\text { integrativa e lições } \\
\text { aprendidas. }\end{array}$ & $\begin{array}{l}\text { Apresentar evidências } \\
\text { científicas sobre fatores } \\
\text { associados ao impacto } \\
\text { ocupacional e psicológico } \\
\text { provocado por elas sobre os } \\
\text { profissionais da saúde. }\end{array}$ & $\begin{array}{l}\text { As condições de trabalho e as próprias } \\
\text { características dos sucessivos fluxos globais } \\
\text { de pandemias revelam desafios de pesquisas } \\
\text { de ordem conceitual e empírica. Novos } \\
\text { processos institucionais são necessários para } \\
\text { otimizar benefícios em termos de saúde } \\
\text { mental e prover o enfrentamento das } \\
\text { situações-problema. Aprender com as ondas } \\
\text { anteriores de pandemias é um passo } \\
\text { importante na definição de uma agenda para } \\
\text { futuras pesquisas. }\end{array}$ \\
\hline A4 & Pereira et al. (2020) & $\begin{array}{l}\text { A pandemia de } \\
\text { COVID-19, o } \\
\text { isolamento social, } \\
\text { consequências na saúde } \\
\text { mental e estratégias de } \\
\text { enfrentamento: uma } \\
\text { revisão integrativa }\end{array}$ & $\begin{array}{l}\text { Realizar uma análise sobre as } \\
\text { consequências na saúde } \\
\text { mental advindas do período } \\
\text { de isolamento social durante } \\
\text { a pandemia de COVID-19e } \\
\text { de prenunciar estratégias de } \\
\text { enfrentamento para } \\
\text { minimizá-las. }\end{array}$ & $\begin{array}{l}\text { Este estudo permitiu refletir sobre a } \\
\text { necessidade de garantir uma comunicação } \\
\text { clara e informativa sobre estratégias para } \\
\text { redução desses sintomas de sofrimento } \\
\text { psíquico, além de fornecer o fundamental } \\
\text { suporte psicológico e social para esses } \\
\text { indivíduos em vulnerabilidade. }\end{array}$ \\
\hline A5 & $\begin{array}{l}\text { Esperdião, Saidel \& } \\
\text { Rodrigues (2020) }\end{array}$ & $\begin{array}{l}\text { Saúde mental: foco nos } \\
\text { profissionais de saúde. }\end{array}$ & $\begin{array}{l}\text { Despertar a atenção da } \\
\text { população, de instituições e } \\
\text { gestores, especialmente nesse } \\
\text { momento da pandemia de } \\
\text { COVID-19. }\end{array}$ & $\begin{array}{l}\text { Neste sentido, como estratégia de cuidado em } \\
\text { saúde mental dos trabalhadores, sugere-se } \\
\text { mapeamento do perfil epidemiológico dos } \\
\text { profissionais em sofrimento psíquico e com } \\
\text { diagnósticos de transtornos mentais. Assim, } \\
\text { essa estratégia direcionaria recursos de } \\
\text { promoção de saúde mental e o bem-estar dos } \\
\text { profissionais de saúde que dispensam sua } \\
\text { força de trabalho em prol do cuidado das } \\
\text { pessoas, muitas vezes para além de suas } \\
\text { possibilidades. }\end{array}$ \\
\hline A6 & $\begin{array}{l}\text { Miranda et al. } \\
\qquad(2020)\end{array}$ & $\begin{array}{l}\text { Condições de trabalho } \\
\text { e o impacto na saúde } \\
\text { dos Profissionais de } \\
\text { enfermagem frente a } \\
\text { covid-19. }\end{array}$ & $\begin{array}{c}\text { Destacar as principais } \\
\text { modificações fisiológicas que } \\
\text { caracterizam o } \\
\text { envelhecimento com ênfase } \\
\text { no sistema nervoso e } \\
\text { relacionar a fisiologia do } \\
\text { envelhecimento à saúde oral } \\
\text { do idoso reconhecendo o } \\
\text { papel da Odontologia na } \\
\text { saúde geral do idoso. }\end{array}$ & $\begin{array}{c}\text { Esta reflexão pode contribuir para repensar a } \\
\text { saúde e segurança dos profissionais de } \\
\text { enfermagem visando uma assistência com } \\
\text { qualidade e segurança aos pacientes frente a } \\
\text { esta doença. }\end{array}$ \\
\hline A7 & $\begin{array}{l}\text { Portugal et al. } \\
\qquad(2020)\end{array}$ & $\begin{array}{l}\text { Percepção do impacto } \\
\text { emocional da equipe de } \\
\text { enfermagem diante da } \\
\text { pandemia de COVID- } \\
\text { 19: relato de } \\
\text { experiência. }\end{array}$ & $\begin{array}{l}\text { Relatar a percepção da equipe } \\
\text { de enfermagem de um } \\
\text { hospital em um município no } \\
\text { interior do estado do } \\
\text { Amazonas, diante da } \\
\text { pandemia de coronavírus, } \\
\text { expondo os principais medos } \\
\text { e anseios frente as incertezas } \\
\text { do novo cenário mundial e os } \\
\text { desafios vindouros. }\end{array}$ & $\begin{array}{l}\text { Os profissionais sofrem as mesmas pressões } \\
\text { psicológicas, principalmente pelo medo } \\
\text { diante da incerteza das condições futuras, } \\
\text { tendo em vista a proporção da pandemia. } \\
\text { Portanto, é preciso que os profissionais de } \\
\text { saúde sejam atendidos em suas inquietações e } \\
\text { desamparos, visto que a regressão da } \\
\text { pandemia depende disso. }\end{array}$ \\
\hline A8 & $\begin{array}{l}\text { Humerez, Ohl \& } \\
\text { Silva (2020) }\end{array}$ & $\begin{array}{l}\text { Saúde mental dos } \\
\text { profissionais de } \\
\text { enfermagem do Brasil } \\
\text { no contexto da } \\
\text { pandemia covid-19: } \\
\text { ação do Conselho } \\
\text { federal de enfermagem. }\end{array}$ & $\begin{array}{l}\text { Refletir sobre a saúde mental } \\
\text { dos profissionais de } \\
\text { enfermagem brasileiros no } \\
\text { contexto da pandemia } \\
\text { COVID-19. }\end{array}$ & $\begin{array}{l}\text { Para atingir esse objetivo, elaborou-se o } \\
\text { projeto de Atendimento de Enfermagem em } \\
\text { Saúde Mental aos profissionais de } \\
\text { enfermagem na pandemia. Depois dos } \\
\text { primeiros trinta dias de atendimentos, os } \\
\text { sentimentos mais emergentes foram: } \\
\text { ansiedade, medo, ambivalência, depressão e } \\
\text { exaustão. }\end{array}$ \\
\hline
\end{tabular}

Fonte: Autores (2020). 
Em relação a saúde mental dos profissionais de Enfermagem na pandemia COVID-19, Humerez, Ohl e Silva (2020) observaram que os trabalhadores da enfermagem pressionados com essa situação apresentam altos níveis de ansiedade, acrescidos do risco de adoecer, provocando severos problemas de saúde mental e aumentando os casos da Síndrome de Burnout, além de gerar ansiedade, depressão e estresse associado.

Corroborando com autor acima, Oliveira et al. (2020) descreveram que a avaliação do impacto psicológico das situações de pandemias revelou associações com quadros de estresse, ansiedade, insônia e sintomatologia depressiva. A saúde mental dos profissionais se mostrou comprometida principalmente pelo medo do próprio contágio e pelo temor de contaminar familiares ou amigos no retorno do trabalho. Verificou-se ainda que os principais fatores relacionados ao impacto ocupacional se referem às mudanças introduzidas na rotina dos profissionais de saúde, como aumento do número de horas de trabalho, criação de espaços de isolamento e incremento das recomendações para uso dos EPI.

Além disso, de acordo com Schmidt et al. (2020) muitos profissionais da saúde no Brasil não têm experiência de atuação em emergências de grande porte, como é o caso da COVID-19, o que representa um estressor adicional. Logo, sugerese a realização de intervenções voltadas à orientação sobre sintomas psicológicos que profissionais da saúde podem apresentar nesse contexto (ex.: estresse, depressão, ansiedade e insônia) bem como estratégias de enfrentamento e autocuidado, gerenciamento de estresse e importância dos momentos de descanso.

Pereira et al. (2020) caracterizam que se deve estar atento para outros fatores de risco, sendo tais, baixa autoestima, diagnóstico prévio de distúrbio mental, baixo poder aquisitivo, falta de suporte social e condições dignas de trabalho. Assim, todos estes fatores podem influenciar significativamente o grau de vulnerabilidade do indivíduo no âmbito psicossocial.

Em uma entrevista realizada com uma equipe médica de um hospital na China, Portugal et al. (2020) relataram que muitos profissionais demonstraram sinais de irritabilidade, angústia e excitabilidade. Informaram que a maior preocupação não era serem infectados, mas sim levar uma possível infecção para suas famílias. Além disso, relataram que a faltam de colaboração por parte dos pacientes estava lhes causando maiores sofrimentos psicológicos, pois muitos não respeitavam as medidas preventivas de disseminação do vírus, consequentemente aumentando as chances de sobrecarregarem as unidades hospitalares. As equipes também informaram suas preocupações quanto a disponibilidade de material de proteção e a sensação de impotência diante de pacientes graves.

Conforme Miranda et al. (2020) é prudente repensar as escalas de trabalho dos PE de modo a diminuir o desgaste físico e emocional. É preciso considerar que a paramentação rigorosa e adequada, bem como a necessidade de economizar EPIs, dificulta a realização de funções fisiológicas como alimentar-se, hidratar-se ou ir ao banheiro, devido ao tempo dispendido para a paramentação e desparamentação entre os procedimentos.

Com base nesse cenário, repercutem-se as estatísticas crescentes de depressão, síndromes variadas de ansiedade, comportamento suicida, síndrome de burnout, surtos psicóticos, uso problemático de álcool e outras drogas, estresse, fadiga e esgotamento profissional. Todas essas situações demonstram o processo de sofrimento e adoecimento mental entre profissionais de saúde, sobretudo na equipe de enfermagem.

Para Esperdião, Saidel e Silva (2020) é iminente o amplo debate para o enfrentamento dessa problemática, cada vez mais próxima de todos nós. Contudo, faz-se indispensável a participação coletiva de todos os atores envolvidos nesse contexto. No eu diz respeito aos trabalhadores, é primordial ampliar seu engajamento nas respectivas entidades de classe e controle social para fortalecer a participação política e aumentar os direitos e as condições de trabalho. Esse posicionamento baseia-se na perspectiva de que o trabalho pode ser um gerador de saúde, à medida em que pode prover ao trabalhador satisfação e bemestar pelo que faz, além de reconhecer o sofrimento e o desgaste mental que o mesmo trabalho propicia. 


\section{Conclusão}

Os profissionais de saúde, que são a linha de frente no combate ao vírus, lidam com o estresse, a pressão psicológica e os riscos da doença cotidianamente. $\mathrm{E}$ os efeitos psicológicos negativos, que podem perdurar por meses após as experiências ruins, são, sobretudo, sintomas de estresse pós-traumático, ansiedade, raiva, medo, irritabilidade e comportamentos evitativos.

Identifica-se que os sintomas psicológicos mais frequentes destacados por este estudo foram primordialmente, o medo, pânico, estresse, ansiedade, culpa e tristeza que ocasionam sofrimento psíquico e podem gerar o aparecimento de transtornos de pânico, transtornos de ansiedade e depressão. Contudo, estes indivíduos devem receber indispensavelmente atenção especial nos cuidados de saúde mental, pois acham-se em situação de vulnerabilidade, onde esses transtornos e sintomas podem ser elevados.

Diante de uma pandemia como a que vivemos, os profissionais da enfermagem constituem parte de um dos grupos mais afetados, expostos a dor emocional e ao risco de contágio que afeta consideravelmente a saúde mental.

Entretanto, cuidados imediatos devem ser aplicados, em todos os níveis e pelas mais distintas áreas de conhecimento, com a finalidade de reduzir resultados ainda mais negativos na saúde mental destes profissionais.

\section{Referências}

Botelho, L. L., Cunha, C. C. A. \& Macedo, M. (2011). O Método da Revisão Integrativa nos estudos Organizacionais. Gestão e Sociedade, 5(11), 121-136.

Brito, F. S. \& Souza, A. P. (2021). O impacto emocional causado pela pandemia do novo coronavírus aos profissionais de enfermagem: Uma revisão integrativa. Research Society and Development, 10(7), e42210716934.

Bezerra, G. D., Sena, A. S. R., Braga, S. T., Santos, M. E. N., Correia, L. F. R., Clementino, K. M. F., Carneiro, Y. V. A. \& Pinheiro, W. R. (2020). O impacto da pandemia por COVID-19 na saúde mental dos profissionais da saúde: revisão integrativa. Revista Enfermagem atual in derme, e020012.

Duarte, M. Q., Santos, M. A. S., Lima, C. P., Giordani, J. P. \& Trentini, C. M. (2020). COVID-19 e os impactos na saúde mental: uma amostra do Rio Grande do Sul. Ciência \& Saúde Coletiva, 25(9), 3401-3411.

Diogo, P. M. J., Sousa, M. O. C. L., Rodrigues, J. R. G. V., Silva, T. A. A. M. A. \& Santos, M. L. F. (2021). Trabalho emocional de enfermeiros da linha de frente do combate à pandemia de COVID-19. Revista Brasileira de Enfermagem, 74(1), e20200660.

Esperidião, E., Saidel, M. G. B. \& Rodrigues, J. (2020). A saúde mental: foco nos profissionais de saúde. Revista Brasileira de Enfermagem, 73(1), e73.

Faro, A., Bahiano, M. A., Nakano, T. C., Reis, C., Silva, B. F. P. \& Vitti, L. S. (2020). COVID-19 e saúde mental: a emergência do cuidado. Estudos psicologia (Campinas), 37, e200074.

Ferreira, D. P., Cardim, M. G. \& Azevedo, M. S. N. (2021). Desafios da gestão de enfermagem na pandemia da COVID-19. Revista científica de enfermagem, 11(34), 364-372.

Gomes, M. P., Barbosa, D. J., Gomes, A. M. T., Souza, F. B. A., Paula, G. S. \& Santo, C. C. E. (2020). Perfil dos profissionais de enfermagem que estão atuando durante a pandemia do novo Coronavírus. Journal of nursing health, 10(4), e20104026.

Humerez, D. C., Ohl, R. I. B. \& Silva, M. C. N. (2020). Saúde mental dos profissionais de enfermagem do Brasil no contexto da pandemia Covid-19: ação do Conselho Federal de Enfermagem. Cogitare enfermagem, 25, e74115.

Kirby, E. E. F., Siqueira, A. S. A., Cunha, D. A. O., Santiago, F. B., Neves, L.M.L. \& Beserra, V. S. (2021). COVID-19 e suas influências psíquicas na percepção da equipe de enfermagem da atenção paliativa oncológica. Revista Mineira de Enfermagem, 25 , e1355.

Miranda, F. M. A., Santana, L. L., Pizzolato, A. C., \& Saquis, L. M. M. (2020). Condições de trabalho e o impacto na saúde dos profissionais de enfermagem frente a Covid-19. Cogitare enfermagem, 25, e72702.

Nunes, B. A. S. (2021). A atuação da liderança de enfermagem como estratégia na prevenção dos impactos da pandemia na saúde mental dos colaboradores. Revista Científica Multidisciplinar Núcleo do Conhecimento, 12, 27-39.

Oliveira, W. A., Oliveira-Cardoso, E. A., Silva, J. L., \& Santos, M. A. (2020). Impactos psicológicos e ocupacionais das sucessivas ondas recentes de pandemias em profissionais da saúde: revisão integrativa e lições aprendidas. Estudos de Psicologia (Campinas), 37 , e200066.

Pereira, M. D., Oliveira, L. C., Costa, C. F. T., Bezerra, C. M. O., Pereira, M. D, Santos, C. K. A. \& Dantas, E. H. M (2020). A pandemia de COVID-19, o isolamento social, consequências na saúde mental e estratégias de enfrentamento: uma revisão integrativa. Revista Research, Society and Development, 9(7), 1-31, e652974548.

Portugal, J. K. A., Reis, M. H.S., Barão, E. J. S., Souza, T. T. G., Guimarães, R. S., Almeida, L. S., Pereira, R.M.O., Freire, N. M., Germano, S. N. F. \& Garrido, M. S. (2020). Percepção do impacto emocional da equipe de enfermagem diante da pandemia de COVID-19: relato de experiência. Revista Eletrônica Acervo Saúde, 46, e3794. 
Research, Society and Development, v. 11, n. 3, e33411321144, 2022

(CC BY 4.0) | ISSN 2525-3409 | DOI: http://dx.doi.org/10.33448/rsd-v11i3.21144

Ramos-Toescher, A. M., Tomaschewisk-Barlem, J. G., Barlem, E. L.D., Castanheira, J. S. \& Toescher, R. L. (2020). Saúde mental de profissionais de enfermagem durante a pandemia de COVID-19: recursos de apoio. Escola Anna Nery, 24, e20200276.

Rosa, T. J. L., Nascimento, S. M., Sousa, R. R. \& Oliveira, D. M. N. (2021). Análise sobre a Saúde Mental dos Profissionais de Enfermagem no enfrentamento da COVID-19: Uma Análise num Hospital Regional. Brazilian Journal of Development, 7(5), 44293-44317.

Schmidt, B., Crepaldi, M. A., Bolze, S. D. A., Neiva-Silva, L., \& Demenech, L. M. (2020). Saúde mental e intervenções psicológicas diante da pandemia do novo coronavírus (COVID-19). Estudos de Psicologia (Campinas), 37, e200063.

Souza, M. T., Silva, M. D. \& Carvalho, R. (2010). Revisão integrativa: o que é e como fazer. Einstein, 8(1), 102-106. 